\title{
Oxidative status and lipid profile in mono- and co-infection with canine monocytic ehrlichiosis")
}

\author{
MEHMET GULTEKIN, KEREM URAL, SERDAR PASA, \\ CANBERK BALIKCI, GAMZE SEVRI EKREN ASICI*

\begin{abstract}
Department of Internal Medicine, *Department of Biochemistry, Faculty of Veterinary Medicine, University of Adnan Menderes, Aydin, Turkey
\end{abstract}

\section{Gultekin M., Ural K., Pasa S., Balikci C., Ekren Asici G. S. Oxidative status and lipid profile in mono- and co-infection with canine monocytic ehrlichiosis Summary}

Vector-borne diseases in dogs are a major health problem of zoonotic importance. Canine monocytic ehrlichiosis (CME) is one of the most common vector-borne infections in dogs. Co-infections with vectorborne diseases are reported to cause more severe pathological effects than mono-infections. The current study investigates the effects of mono- and co-infections with CME on oxidative status and lipid profiles in dogs. Dogs with vector-borne diseases were divided into two groups, mono-infected $(n=22)$ and co-infected $(n=23)$ with $\mathrm{CME}$, and compared to a healthy group $(n=20)$. Double and triple co-infections with other vector-borne diseases included A. phagocytophilum, $L$. infantum, and D. immitis. The serum lipid profile (high-density lipoprotein (HDL), low-density lipoprotein (LDL), cholesterol, and triglycerides) and the total and individual markers of oxidative status (total antioxidant capacity (TAC), total oxidant status (TOS), malondialdehyde (MDA), paraoxonase (PON-1), and arylesterase (ARE)) were evaluated. Significant decreases in PON-1 and ARE enzyme activities and HDL concentration, as well as increases in TOS level, MDA, LDL, and triglyceride concentrations were determined in both mono- and co-infected groups compared to the healthy control group. No significant differences between the groups were found in the TAC level or cholesterol concentration. In conclusion, alterations in lipid profile and increase in oxidative stress were observed in dogs with CME compared to the healthy control group, however, no significant differences were detected between mono- and co-infected dogs.

Keywords: canine monocytic ehrlichiosis, oxidative status, lipid profile, co-infection

Canine monocytic ehrlichiosis (CME) is an important vector-borne disease in dogs worldwide. Ehrlichia canis, a Gram-negative, obligate intracellular bacterium is the etiological agent of the disease and is transmitted to dogs by Rhipicephalus sanguineus (28). Infection may result in acute, subclinical, or chronic stages of the disease with a variety of clinical and laboratory symptoms. The acute stage mostly presents with fever, anorexia, weight loss, lethargy, lymphadenomegaly, hemorrhagic disorders, anemia, thrombocytopenia, and leukocytosis or leukopenia. Dogs may recover from the acute stage with treatment, remain subclinically infected, or progress to a chronic stage with more severe symptoms than acute findings $(15,33)$.

Oxidative imbalances may occur in the organism as the disease progresses. Oxidative stress and increased lipid peroxidation has been shown in dogs with $\mathrm{CME}$ because of free-radical activity and an impaired antioxidant system $(2,8,36)$. Paraoxonase $(\mathrm{PON}-1)$ and

1) This study was partially summarized from the project funded by Adnan Menderes University Research Projects Funding Unit with project number VTF-16003. arylesterase (ARE) are esterase enzymes with antioxidant properties in a complementary structure. PON-1 shows a structure that binds to high-density lipoprotein (HDL) and protects low-density lipoprotein (LDL) and HDL from oxidative stress by contributing to the destruction of organophosphorus compounds, such as paraoxone and lipid peroxidation products (11). In humans, PON-1 has been shown to play a role in the pathophysiology of many diseases (23). Besides being a biomarker of the antioxidant status, PON-1 is also evaluated as a negative acute phase protein in humans and dogs $(31,33)$. Decreased activity of PON-1 in leishmaniasis, babesiosis, acute pancreatitis, enteritis, and CME in dogs has been reported $(16,17,19,24$, $31,33,39)$.

Dogs are reservoir hosts for a wide spectrum of vector-borne pathogens $(9,28)$. Although dogs may be infected with a single pathogen, multiple vector-borne diseases can occur sequentially or simultaneously when dogs are exposed to vectors infected with different organisms (4). Co-infections with vector-borne 
diseases are reported to cause more severe pathological effects than mono-infections $(9,14)$. However, a large proportion of studies in dogs have been carried out by evaluating vector-borne diseases separately. Moreover, to the best of the author's knowledge, no studies have been conducted to evaluate changes in lipid profiles of dogs with CME. Therefore, this study aims to investigate the effects of mono- and co-infections with $\mathrm{CME}$ on oxidative status and lipid profiles in dogs.

\section{Material and methods}

Animal population and sample collection. The present study was performed at the University of Adnan Menderes, Faculty of Veterinary, Department of Internal Medicine, located in the Aegean region of Turkey from October 2015 to December 2016. The study protocol was approved by the University of Adnan Menderes HADYEK unit, institutional laboratory animals ethics committee (no: 2015/122, 27.10.2015). Informed written consent was obtained from all owners prior to enrolment.

A total of 65 dogs were enrolled: 45 mono- and coinfected with CME, and 20 sex- and age-matched, healthy control dogs. The initial diagnosis of vector-borne diseases was established in dogs presenting one or more clinical signs associated with the disease. Commercial rapid test kits (Snap 4Dx, Snap Leishmania, Idexx, USA) with the enzyme-linked immunosorbent assay principle were used for the simultaneous qualitative detection of canine antibodies to E. canis, A. phagocytophilum, B. burgdorferi, L. infantum, and the $D$. immitis antigen in serum samples. In addition, babesiosis and hepatozoonosis were evaluated through the cytological examination of blood samples. Dogs that were previously diagnosed with vector-borne diseases or medically treated were not included in the study. The control group was classified as healthy on the basis of history, clinical examination, and laboratory tests. Blood samples were obtained from Vena cephalica antebrachii following an overnight fasting, and transferred into tubes containing EDTA for complete blood counts and into plain tubes to obtain serum through centrifugation at $3000 \mathrm{rpm}$ for 15 minutes. Serum aliquots were evaluated for hemolysis and lipemia and then stored at $-20^{\circ} \mathrm{C}$ until analysis.

Haematological and biochemical analysis. Erythrocyte, leukocyte, and thrombocyte counts, and the hematocrit value were measured with a complete blood count device (Abacus Junior Vet 5, Hungary). Serum samples were used for the estimation of the biochemical profile with an automated clinical chemistry analyzer (Chemray 120 Automated Analyzer, Rayto, China). Total antioxidant capacity groups
(TAC) and total oxidant status (TOS) levels were measured with commercially available test kits (Rel Assay Diagnostics, Turkey) and by methods described by Erel $(12,13)$. The activities of PON-1 and ARE (Rel Assay Diagnostics, Turkey), as well as concentrations of HDL, LDL, cholesterol, and triglyceride (Archem Diagnostics, Turkey) were also measured with commercial test kits. The malondialdehyde (MDA) concentration, to determine the lipid peroxidation, was measured on a spectrophotometer (Shimadzu, Japan) by a method described by Yoshoiko et al. (41). All biochemical analyses used in this study had intra-run coefficients with a variation $<10 \%$.

Statistical analysis. Statistical analysis was carried out with a statistical software package (SPSS 22.0, SPSS Inc., Chicago, USA). One-way analysis of variance (ANOVA) was used for the comparison and the Tukey test was used for post hoc analysis to determine differences in the biochemical parameters of the groups. GraphPad Prism (GraphPad Software Inc., La Jolla, USA) was used to plot graphs. Risk ratios were calculated for clinical and haematological abnormalities in mono- and co-infected groups. A 95\% confidence interval was calculated for the risk ratios. Probability values of $\mathrm{P}<0.05$ were considered significant.

Tab. 1. Clinical and haematological abnormalities in mono- and co-infected

\begin{tabular}{|c|c|c|c|c|c|c|}
\hline \multirow{2}{*}{$\begin{array}{l}\text { Clinical and } \\
\text { haematological } \\
\text { abnormalities }\end{array}$} & \multicolumn{2}{|c|}{$\begin{array}{l}\text { Mono-infected } \\
\quad(\mathrm{n}=22)\end{array}$} & \multicolumn{2}{|c|}{$\begin{array}{l}\text { Co-infected } \\
(\mathrm{n}=23)\end{array}$} & \multicolumn{2}{|c|}{$\begin{array}{l}\text { Risk analysis between } \\
\text { mono- and co-infected dogs }\end{array}$} \\
\hline & Positive (n) & $\%$ & Positive (n) & $\%$ & Risk ratio (95\% Cl) & $\mathbf{P}$ \\
\hline Lymphadenopathy & 20 & 90 & 16 & 69 & $1.30(0.96-1.76)$ & 0.07 \\
\hline Anorexia & 15 & 68 & 11 & 47 & $1.42(0.85-2.38)$ & 0.16 \\
\hline Fever & 14 & 63 & 8 & 34 & $1.83(0.96-3.47)$ & 0.05 \\
\hline Weight loss & 8 & 36 & 12 & 52 & $0.69(0.35-1.37)$ & 0.28 \\
\hline Lethargy & 8 & 36 & 13 & 56 & $0.64(0.33-1.24)$ & 0.17 \\
\hline Dermatologic lesions & 4 & 18 & 12 & 52 & $0.34(0.13-0.91)$ & 0.01 \\
\hline Epistaxis & 3 & 13 & 6 & 26 & $0.52(0.14-1.83)$ & 0.29 \\
\hline Anemia & 16 & 72 & 19 & 82 & $0.88(0.64-1.20)$ & 0.42 \\
\hline Thrombocytopenia & 15 & 68 & 17 & 73 & $0.99(0.63-1.34)$ & 0.67 \\
\hline Leukocytosis & 10 & 45 & 7 & 30 & $1.49(0.69-3.22)$ & 0.29 \\
\hline Leukopenia & 3 & 13 & 6 & 26 & $0.52(0.14-1.83)$ & 0.29 \\
\hline Pancytopenia & 2 & 2 & 6 & 26 & $0.34(0.07-1.54)$ & 0.13 \\
\hline
\end{tabular}

Tab. 2. Oxidative status and lipid profiles in mono-infected, co-infected and healthy groups (Mean \pm SD)

\begin{tabular}{|l|c|c|c|c|}
\hline \multicolumn{1}{|c|}{ Parameters } & $\begin{array}{c}\text { Healthy } \\
(\mathrm{n}=20)\end{array}$ & $\begin{array}{c}\text { Mono-infected } \\
(\mathrm{n}=22)\end{array}$ & $\begin{array}{c}\text { Co-infected } \\
(\mathrm{n}=23)\end{array}$ & $P$ \\
\hline HDL $(\mathrm{mg} / \mathrm{dL})$ & $139.5 \pm 14.1$ & $71.5 \pm 18.6$ & $77.9 \pm 22.0$ & 0.000 \\
LDL $(\mathrm{mg} / \mathrm{dL})$ & $47.6 \pm 27.9$ & $114.9 \pm 49.8$ & $112.9 \pm 45.3$ & 0.000 \\
\hline CHOL $(\mathrm{mg} / \mathrm{dL})$ & $195.1 \pm 25.3$ & $202.4 \pm 56.4$ & $201.2 \pm 53.7$ & 0.906 \\
TRIG $(\mathrm{mg} / \mathrm{dL})$ & $40.0 \pm 6.6$ & $79.6 \pm 41.1$ & $68.6 \pm 28.3$ & 0.002 \\
\hline PON-1 (U/L) & $255.7 \pm 43.0$ & $157.1 \pm 61.9$ & $173.3 \pm 52.8$ & 0.000 \\
ARE $(\mathrm{U} / \mathrm{L})$ & $281.8 \pm 55.8$ & $201.2 \pm 48.0$ & $195.0 \pm 67.7$ & 0.000 \\
TAC $(\mathrm{mmol}$ Trolox Equiv./L) & $0.66 \pm 0.01$ & $0.65 \pm 0.05$ & $0.63 \pm 0.05$ & 0.165 \\
TOS $\left(\mu \mathrm{mol} \mathrm{H} \mathrm{H}_{2}\right.$ Equiv./L) & $6.6 \pm 0.9$ & $17.2 \pm 9.4$ & $19.0 \pm 11.1$ & 0.000 \\
\hline MDA $(\mu \mathrm{mol} / \mathrm{L})$ & $17.7 \pm 2.8$ & $34.3 \pm 13.9$ & $35.7 \pm 15.1$ & 0.000 \\
\hline
\end{tabular}




\section{Results and discussion}

Among the 45 dogs with vector-borne diseases, 22 were mono-infected and 23 were co-infected with CME. Co-infections with another vector-borne disease included A. phagocytophilum $(\mathrm{n}=7)$, L. infantum $(\mathrm{n}=6)$, and $D$. immitis $(\mathrm{n}=2)$. Six dogs were co-infected with both $A$. phagocytophilum and L. infantum, and two dogs were co-infected with $A$. phagocytophilum and $D$. immitis. Twenty dogs were classified as healthy on the basis of their history, clinical examination, and laboratory tests. The mean ages $( \pm \mathrm{SD})$ of the healthy, mono-infected, and co-infected groups were $4.3( \pm 2.6)$, $4.7( \pm 2.8)$, and $4.8( \pm 2.7)$ years, respectively. There were 11 males and 9 females in the healthy group, 10 males and 12 females in the mono-infected group, and 12 males and 11 females in the co-infected group. Age, sex, and body weight differences between the groups were insignificant.

Clinical and haematological abnormalities that presented in more than one case in mono- and co-infected groups are summarized in Tab. 1. Dermatological lesions were found to be more common in the co-infected group $(\mathrm{p}<$ $0.05)$, but no other significant differences were observed.

The oxidative status and lipid profiles of healthy, monoinfected, and co-infected dogs are presented in Tab. 2. Significant decreases in PON-1 and ARE enzyme activities and HDL concentration, and increases in TOS level, MDA, LDL, and triglyceride concentrations were found in both mono-infected and co-infected groups compared to the control group. However, no significant differences were detected between mono-infected and co-infected dogs compared to the control group. There were also no significant differences between the groups in the TAC level and cholesterol concentration. The levels of parameters with significant differences between the groups are presented as graphs in Fig. 1.

Vector-borne diseases in dogs are a major health problem of zoonotic importance. One of the most common vector-borne infections in dogs is CME (28). Seroprevalence rates of CME in dogs range between 1 and $41.5 \%$ in different areas of Turkey (38). The present study was conducted in Aydin, in the
Aegean Region of the country, where vector-borne diseases are relatively more prevalent (40). Some vectors, especially ticks, can act as vectors for more than one infectious agent, causing co-infections (3). Co-infection with canine vector-borne diseases appears to be common in endemic regions and potentially complicates the diagnosis and management of cases $(9,14)$. The risk of acquiring vector-borne co-infections may depend on the prevalence of multi-infected vectors. $R$. sanguineus appears to be the main vector for $E$. canis, whereas the only known vector for A. phagocytophilum is Ixodes ricinus in Europe (35). Transmission of $L$. infantum can occur from the bite of sand flies, whereas most mosquito species are capable of transmitting $D$. immitis (28). In the present study, among the 45 dogs with vector-borne diseases, 22 and 23 were mono- and co-infected with CME, respectively. Among these, eight triple and 15 double co-infection cases were detected. The most frequent co-infection was with $A$. phagocytophilum $(\mathrm{n}=13)$, followed by L. infantum $(\mathrm{n}=12)$, and D. immitis $(\mathrm{n}=3)$. These results draw attention to the relatively
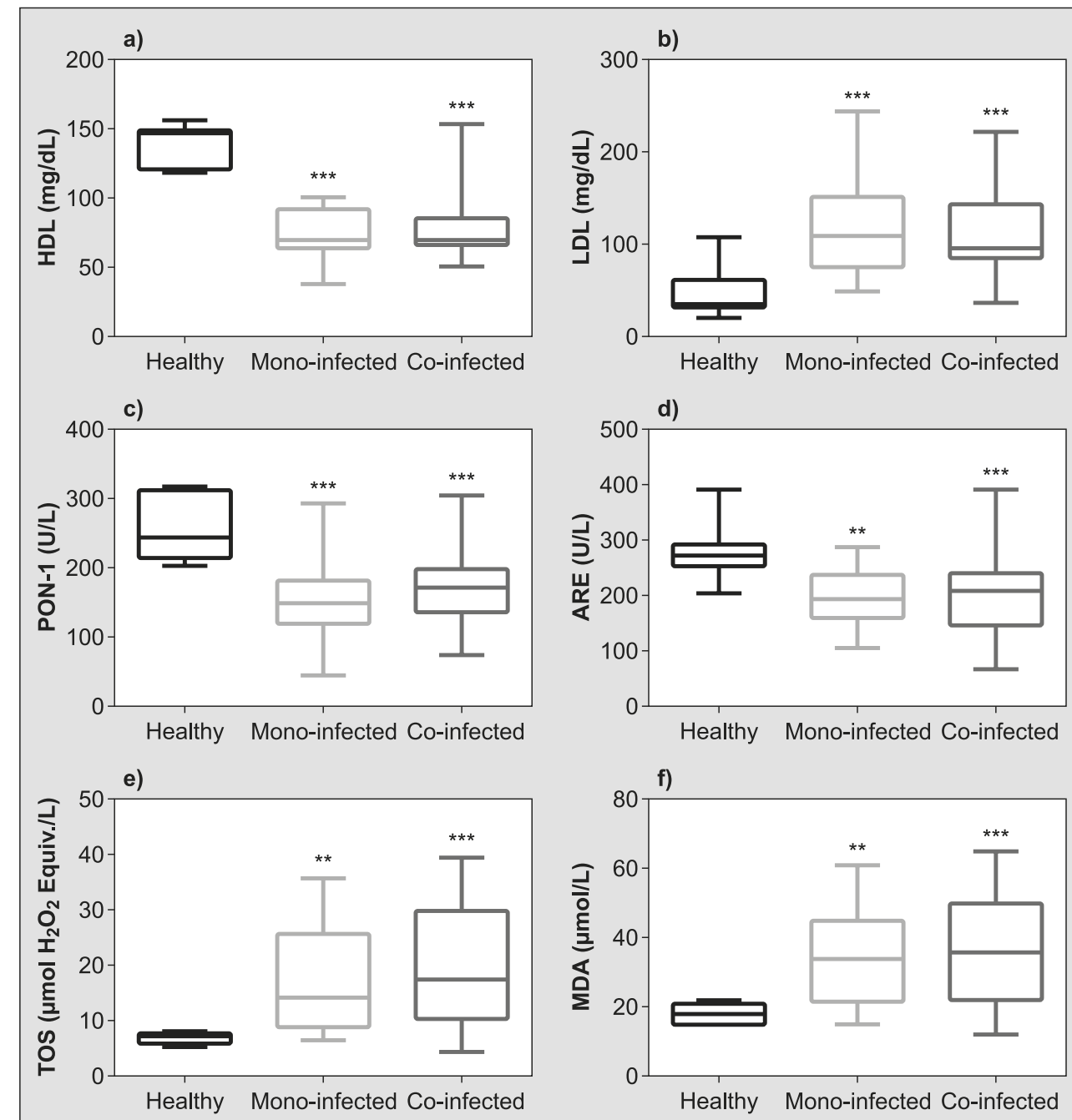

Fig. 1. a)-f) The levels of the parameters with significant differences between the groups are presented as box and whiskers plots

Explanations: Boxes span the $25^{\text {th }}$ to the $75^{\text {th }}$ percentile; whiskers show the $10^{\text {th }}$ and $90^{\text {th }}$ percentiles; the line inside each box denotes the median. The data were analysed using ANOVA test followed by Tukey's post hoc test. Asterisks denote pairwise comparisons to healthy controls. $* \mathrm{P}<0.05, * * \mathrm{P}<0.01, * * * \mathrm{P}<0.001$ 
high prevalence of various vectors and vector-borne infections in the region.

At least one clinical symptom was present in all monoand co-infected dogs. The most common abnormalities in both groups, without any significant difference, included lymphadenopathy, anorexia, fever, weight loss, lethargy, and epistaxis as clinical findings, as well as anemia, thrombocytopenia, leukocytosis/leukopenia, and pancytopenia as haematological changes. These findings were as expected in dogs infected with CME and other vector-borne diseases $(29,35)$. However, dermatological lesions were found to be more common in the co-infected group $(\mathrm{p}<0.05)$. This condition may have been related the co-infection with L. infantum.

Oxidative damage is implicated in the pathophysiology of many infectious and non-infectious conditions. There are few studies related to the effects of oxidative damage on the pathophysiology of CME. Kumar et al. (21) investigated lipid peroxidation in dogs infected with CME and co-infected with babesiosis, finding no significant difference between healthy dogs and dogs with CME, whereas higher concentrations of MDA were found in dogs with co-infections. Increases in levels of nitric oxide, lipid peroxidation, and protein oxidation have been shown in dogs experimentally infected with CME (8). Higher levels of lipid peroxidation and decreased activities of superoxide dismutase, glutathione, and catalase have been shown in natural co-infections with CME, babesiosis, anaplamosis, and hepatozoonosis (36). Bottari et al. (2) found higher levels of advanced oxidation protein products and the ferric-reducing ability of plasma in acute and subclinical phases of CME. The present study aims to represent the oxidative status with total and individual parameters and to show their relationship to the lipid profile. Methods for determining the oxidative status include direct and indirect measurements of oxidants and antioxidants. Despite some disadvantages, measurements of TAC and TOS concentrations are a practical way to identify the oxidative status of an organism $(13,32)$. One of the products formed during the oxidative damage-induced decomposition of polyunsaturated fatty acid is MDA (8). The present study found significant increases in TOS levels and MDA concentrations in both monoand co-infected dogs. Moreover, no significant difference between the groups was found in the TAC level. These results suggest the presence of oxidative damage and lipid peroxidation in dogs mono- and co-infected with CME.

The enzymes PON-1 and ARE are part of an antioxidant system that protects HDL and LDL particles from oxidation $(11,23)$. The significantly decreased activity of PON-1 has been demonstrated in dogs diagnosed with leishmaniosis, babesiosis, parvoviral enteritis, and acute pancreatitis $(17,24,31,39)$. Furthermore, PON-1 has been evaluated in dogs experimentally infected with CME (16, 33). Rudoler et al. (33) evaluated PON-1 activity as part of antioxidant response following the experimental infection of vaccinated and non-vaccinated dogs. In the present study, decreased activity of PON-1 was shown in groups, but changes among groups were indistinguishable. Karnezi et al. (16) examined PON-1 activity in dogs experimentally infected with $\mathrm{CME}$ for an assessment of responses to rifampicin treatment over 42 days. Throughout the study, PON-1 activity did not differ between treated and untreated dogs. A decreased activity of PON-1 was found in only one of the groups, on day 14 after inoculation (16). Another esterase enzyme, ARE has lipophilic antioxidant characteristics and acts in conjunction with PON-1. However, the activity of ARE in dogs has been studied less than that of PON-1. In the present study, PON1 and ARE activities were decreased in both mono-infected and co-infected groups. Changes in the activity of PON-1 were consistent with results from Rudoler et al. (33). While the individual antioxidants - PON-1 and ARE activities - were found to be significantly decreased in the current study, no significant differences were found in the TAC value, representing the overall antioxidant characteristics of the organism. This may be attributed to the enhancement of other enzymatic or non-enzymatic antioxidants to compensate for the effects of oxidative damage $(6,18)$.

Lipids are one of the substrates most susceptible to oxidative damage. To the best of the current authors' knowledge, this is the first report on the lipid profile in dogs with CME. According to the results of the study, the serum lipid profile significantly changes in dogs naturally mono- and co-infected with CME.

Ehrlichiosis is known as a catabolic disease - like most haemoparasites, the agent of the disease cannot synthesize its own lipids - and is predominantly bound to HDL derived from the host's plasma $(20,22)$. In a present study, HDL values were significantly lower in both groups infected with vector-borne diseases compared to healthy dogs. Lower concentrations of HDL may be associated with the consumption of the host's HDL by $E$. canis, the decreased activity of lipoprotein lipase, or the impaired function of the liver resulting from the effects of vector-borne diseases $(25,26)$. These results are consistent with previous findings in dogs with babesiosis $(7,25)$ and visceral leishmaniasis (10). Higher concentrations of triglycerides were found in mono- and co-infected groups. Increased levels of triglycerides have been demonstrated as part of the host's response to infection (5) and presented in dogs with babesiosis (25). Moreover, a defect in the removal of triglycerides from circulation, or increased hepatic production, may be associated with the increase (25). Bacteria incorporate the cholesterol derived from host cells to support membrane integrity; therefore, cholesterol has become necessary for their survival and infection (22). Nevertheless, the production or biosynthetic modification of cholesterol enzymes was not identified in $E$. canis. No significant difference in cholesterol level was found between groups in the current study. It can be suggested that a host cannot affect to the increased intracellular cholesterol to sustaining intracellular cholesterol with cholesterol-mediated feedback regulation, 
similar to cells infected with A. phagocytophilum (30). An increased concentration of serum LDL - the "bad lipoprotein" - is known as atherogenic (1). In the present study, LDL concentrations were found to be higher in both mono-infected and co-infected groups compared to healthy dogs, similar to dogs with leishmaniasis (10, 27, 34). In humans, PON-1 deficiency has been reported as a predisposing factor for atherosclerosis and cardiovascular disease (23). Additionally, decreased levels of HDL and increased levels of LDL and triglycerides are referred to as an atherogenic lipid profile in humans (37), as observed in the current study. These findings suggest the importance of further studies on the cardiovascular effects of CME.

In conclusion, alterations in lipid profile and increase in oxidative stress were observed in dogs with CME compared to the healthy control group, however, no significant differences were detected between monoand co-infected dogs. The decrease in PON-1 and ARE activities and in HDL concentration and the increase in LDL concentration and lipid peroxidation may increase the complications of vector-borne diseases. These findings should be taken into account in the diagnosis, prognosis, and treatment of the cases. Further sequential studies of experimental infections are necessary to gather more knowledge on the pathogenesis of co-infections with vector-borne diseases.

\section{References}

1. Bauer J. E: Lipoprotein-mediated transfer of dietary and synthesized lipids and lipid abnormalities of dogs and cats. J. Am. Vet. Med. Assoc. 2004, 224 668-675

2. Bottari N. B., Crivellenti L. Z., Borin-Crivellenti S., Oliveira J. R., Coelho S. B., Contin C. M. Tatsch E. Moresco R. N. Santana A. E. Tonin A. A., TinucciCosta $M$. : Iron metabolism and oxidative profile of dogs naturally infected by Ehrlichia canis: Acute and subclinical disease. Microb. Pathog. 2016, 92, 26-29.

3. Cardoso L., Mendão C., de Carvalho L. M.: Prevalence of Dirofilaria immitis, Ehrlichia canis, Borrelia burgdorferi sensu lato, Anaplasma spp. and Leishmania infantum in apparently healthy and CVBD-suspect dogs in Portugal - a national serological study. Parasit. Vectors 2012, 5, 62

4. Cardoso L., Yisaschar-Mekuzas Y., Rodrigues F. T., Costa Á., Machado J., DizLopes D., Baneth G.: Canine babesiosis in northern Portugal and molecular characterization of vector-borne co-infections. Parasit. Vectors 2010, 3, 27.

5. Carpentier Y. A., Scruel O.: Changes in the concentration and composition of plasma lipoproteins during the acute phase response. Curr. Opin. Clin. Nutr. Metab. Care 2002, 5, 153-158.

6. Chaudhuri S., Varshney J. P., Patra R. C.: Erythrocytic antioxidant defense, lipid peroxides level and blood iron, zinc and copper concentrations in dogs naturally infected with Babesia gibsoni. Res. Vet. Sci. 2008, 85, 120-124.

7. Cunha B. A., Crean J., Rosenbaum G.: Lipid abnormalities in babesiosis. Am. J. Med. 2000, 108, 758-759.

8. Da Silva A. S., Tonin A. A., Thorstenberg M. L., Leal D. B., Fighera R., Flores $M$. M., Lopes $S$. T.: Relationship between butyrylcholinesterase activity and liver injury in mice acute infected with Toxoplasma gondii. Pathol. Res. Pract. 2013, 209, 95-98.

9. De Tommasi A. S., Otranto D., Dantas-Torres F., Capelli G., Breitschwerdt E. B., de Caprariis D.: Are vector-borne pathogen co-infections complicating the clinical presentation in dogs? Parasit. Vectors 2013, 6, 97.

10. Durgut R., Dalkilinc D., Güzel M.: Evaluation of the serum lipid profiles in dogs with symptomatic Visceral Leishmaniasis. Kafkas University of Veterinary Fak. Derg. 2012, 18, 585-588.

11. Elkiran E. T., Mar N., Aygen B., Gursu F., Karaoglu A., Koca S.: Serum paraoxonase and arylesterase activities in patients with lung cancer in a Turkish population. BMC Cancer 2007, 7, 48.

12. Erel O.: A novel automated direct measurement method for total antioxidant capacity using a new generation, more stable ABTS radical cation. Clin. Biochem. 2004, 37, 277-285

13. Erel $O$.: A new automated colorimetric method for measuring total oxidant status. Clin. Biochem. 2005, 38, 1103-1111.

14. Gaunt S., Beall M., Stillman B., Lorentzen L., Diniz P., Chandrashekar R. Breitschwerdt E.: Experimental infection and co-infection of dogs with Anaplasma platys and Ehrlichia canis: hematologic, serologic and molecular findings. Parasit. Vectors 2010, 3, 33.
15. Harrus S., Waner T: Diagnosis of canine monocytotropic ehrlichiosis (Ehrlichia canis): an overview. Vet. J. 2011, 187, 292-296.

16. Karnezi D., Ceron J. J., Theodorou K., Leontides L., Siarkou V. I., Martinez S. Tvarijonaviciute A., Harrus S., Koutinas C. K., Pardali D., Mylonakis M. E. Acute phase protein and antioxidant responses in dogs with experimental acute monocytic ehrlichiosis treated with rifampicin. Vet. Microbiol. 2016, 184, 59-63.

17. Kocaturk M., Tvarijonaviciute A., Martinez Subiela S., Tecles F., Eralp O., Yilmaz Z., Ceron J. J.: Inflammatory and oxidative biomarkers of disease severity in dogs with parvoviral enteritis. J. Small Anim. Pract. 2015, 56, 119-124.

18. Kogika M. M., Lustoza M. D., Hagiwara M. K., Caragelasco D. S., Martorelli C. R., Mori C. S.: Evaluation of oxidative stress in the anemia of dogs with chronic kidney disease. Vet. Clin. Pathol. 2014, 44, 70-78.

19. Kuleš J., de Torre-Minguela C., Rafaj R. B., Gotić J., Nižić P., Ceron J. J., Mrljak V.: Plasma biomarkers of SIRS and MODS associated with canine babesiosis. Res. Vet. Sci. 2016, 105, 222-228.

20. Kuleš J., Mrljak V., Rafaj R. B., Selanec J., Burchmore R., Eckersall P. D. Identification of serum biomarkers in dogs naturally infected with Babesia canis canis using a proteomic approach. BMC Vet. Res. 2014, 10, 111.

21. Kumar A., Varshney J. P., Patra R. C.: A comparative study on oxidative stress in dogs infected with Ehrlichia canis with or without concurrent infection with Babesia gibsoni. Vet. Res. Commun. 2006, 30, 917-920.

22. Lin M., Rikihisa Y.: Ehrlichia chaffeensis and Anaplasma phagocytophilum lack genes for lipid A biosynthesis and incorporate cholesterol for their survival. Infect. Immun. 2003, 71, 5324-5331.

23. Litvinov D., Mahini H., Garelnabi M.: Antioxidant and anti-inflammatory role of paraoxonase 1: implication in arteriosclerosis diseases. N. Am. J. Med. Sci. 2012, 4, 523-532

24. Martinez-Subiela S., Cerón J. J., Strauss-Ayali D., Garcia-Martinez J. D. Tecles F., Tvarijonaviciute A., Caldin M. Baneth G.: Serum ferritin and paraoxonase-1 in canine leishmaniosis. Comp. Immunol. Microbiol. Infect. Dis. 2014, 37, 23-29.

25. Mrljak V., Kučer N., Kuleš J., Tvarijonaviciute A., Brkljačić M., Crnogaj M., Rafaj R. B.: Serum concentrations of eicosanoids and lipids in dogs naturally infected with Babesia canis. Vet. Parasitol. 2014, 201, 24-30.

26. Nakamura T., Reicher H., Sattler $W$ : Comparison of RRR- $\alpha$-and all-rac- $\alpha-$ -tocopherol uptake by permanent rat skeletal muscle myoblasts (L6 cells): effects of exogenous lipoprotein lipase. Lipids 1998, 33, 1001-1008.

27. Nieto C. G., Barrera R., Habela M. A., Navarrete I., Molina C., Jiménez A., Serrera J. L.: Changes in the plasma concentrations of lipids and lipoprotein fractions in dogs infected with Leishmania infantum. Vet. Parasitol. 1992, 44, $175-182$

28. Otranto D., Dantas-Torres F., Breitschwerdt E. B.: Managing canine vector-borne diseases of zoonotic concern: part one Trends Parasitol. 2009a, 25, 157-163.

29. Otranto D., Dantas-Torres F., Breitschwerdt E. B.: Managing canine vector-borne diseases of zoonotic concern: part two. Trends Parasitol. 2009b, 25, 228-235.

30. Rikihisa Y.: Anaplasma phagocytophilum and Ehrlichia chaffeensis: subversive manipulators of host cells. Nat. Rev. Microbiol. 2010, 8, 328-339.

31. Rossi G., Ibba F., Meazzi S., Giordano A., Paltrinieri S.: Paraoxonase activity as a tool for clinical monitoring of dogs treated for canine leishmaniasis. Vet. J. 2014, 199, 143-149.

32. Rubio C. P., Hernández-Ruiz J., Martinez-Subiela S., Tvarijonaviciute A., Ceron $J$. J.: Spectrophotometric assays for total antioxidant capacity (TAC) in dog serum: an update. BMC Vet. Res. 2016, 12, 166.

33. Rudoler N., Harrus S., Martinez-Subiela S., Tvarijonaviciute A., van Straten M. Cerón J. J., Baneth G.: Comparison of the acute phase protein and antioxidant responses in dogs vaccinated against canine monocytic ehrlichiosis and naive-challenged dogs. Parasit. Vectors 2015, 8, 175.

34. Ruiz-Tapia P., Zaragoza-Bayle C., Duque-Carrasco F. J., Barrera-Chacón R. Changes in plasma lipid profile in dogs with cutaneous diseases. Rev. Cient-Fac. Cien. V. 2014, 24, 502-508.

35. Sainz Á., Roura X., Miró G, Estrada-Peña A., Kohn B., Harrus S., SolanoGallego L.: Guideline for veterinary practitioners on canine ehrlichiosis and anaplasmosis in Europe. Parasit. Vectors 2015, 8, 75

36. Sarma K., Mondal D., Saravanan M., Mahendran K.: Evaluation of haemato-biochemical and oxidative indices in naturally infected concomitant tick borne intracellular diseases in dogs. Asian Pac. J. Trop. Dis. 2015, 5, 60-66.

37. Siewert S., Gonzalez I. I., Lucero R. O., Ojeda M. S.: Association of cholesteryl ester transfer protein genotypes with paraoxonase 1 activity, lipid profile and oxidative stress in type 2 diabetes mellitus: A study in San Luis, Argentina. J. Diabetes Investig. 2015, 6, 67-77.

38. Tunc H. O., Aktas M. S.: Tick-Borne Diseases in Dogs in Turkey. Erciyes Univ. Vet. Fak. Derg. 2016, 13, 223-230.

39. Tvarijonaviciute A., García Martínez J. D., Caldin M., Martínez-Subiela S. Tecles F., Pastor J., Ceron J. J.: Serum paraoxonase 1 (PON1) activity in acute pancreatitis of dogs. J. Small Anim. Pract. 2015, 56, 67-71.

40. Ural K., Gultekin M., Atasoy A., Ulutas B.: Spatial distribution of vector borne disease agents in dogs in Aegean region, Turkey. Rev. MVZ Córdoba 2014, 19, 4086-4098

41. Yoshoiko T., Kawada K., Shimada T.: Lipid peroxidation in maternal and cord blood and protective mechanism against activated-oxygen toxicity in the blood. Am. J. Obstet. Gynecol. 1979, 135, 372-376.

Corresponding author: Mehmet Gultekin, DVM, PhD; Department of Internal Medicine, Faculty of Veterinary Medicine, Adnan Menderes University, Isikli, Aydın, 09017, Turkey; e-mail: gultekinmehmet@gmail.com 